\title{
An Estimation Method for Environmental Friction Based on Body Dynamic Model of Caenorhabditis elegans
}

\author{
Zu Soh \\ Department of System Cybernetics, Hiroshima University, 1-4-1 Kagamiyama \\ Higashi-Hiroshima, Hiroshima 739-8527, Japan \\ Michiyo Suzuki \\ Department of Radiation-Applied Biology, National Institutes for Quantum and Radiological Science and Technology, \\ 1233 Watanuki, Takasaki, Gunma 370-1292, Japan \\ Toshio Tsuji \\ Department of System Cybernetics, Hiroshima University, 1-4-1 Kagamiyama \\ Higashi-Hiroshima, Hiroshima 739-8527, Japan \\ E-mail:sozu@bsys.hiroshima-u.ac.jp
}

\begin{abstract}
Caenorhabditis elegans is a small worm which is approximately $1 \mathrm{~mm}$ in length. The present study proposes an estimation method for frictional force using locomotion information obtained from video analysis of actual worms. The results indicate that the body model driven by the estimated frictional force can trace the locomotion of the worm within a low error level of $4 \%$ of the body length. The proposed method can be applied to analyze the relationship between friction and gait control.

Keywords: Caenorhabditis elegans, frictional force, dynamics model, video analysis, locomotion
\end{abstract}

\section{Introduction}

Caenorhabditis elegans is a small soil-dwelling worm with a slender translucent body composed of around 1,000 cells. It is approximately $1 \mathrm{~mm}$ in length and about $5.0 \mu \mathrm{g}$ in weight (Figure 1(a)). Its neural network, which is composed of only 302 neurons, allows the processing of environmental information such as temperature and chemical gradients so that the worm can act in response to its surroundings. Thanks to the small scale of its neural network, C. elegans has become a favored model organism for the analysis of neuronal mechanisms underlying information processing, especially since White et al. revealed a connective structure between its neurons and muscle cells. ${ }^{1}$

In this context, the mechanism of gait control is an interesting target among various life phenomena because commands generated by neural networks can be easily observed through locomotion. Accordingly, the mechanisms of information processing in C. elegans can 


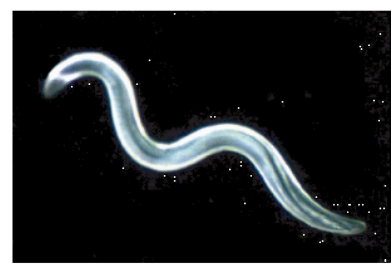

(a) C. elegans

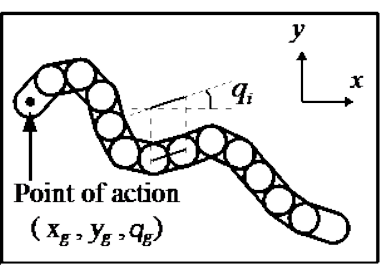

(b) Body Model

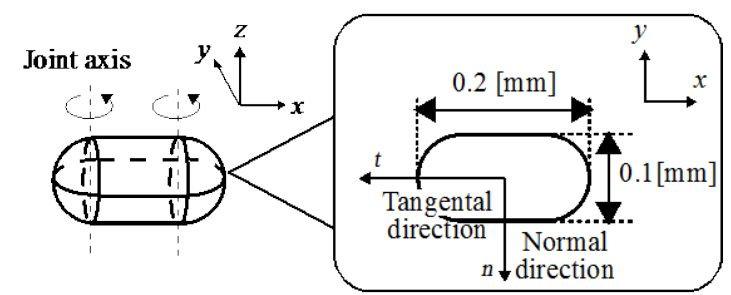

(c) Model of each link

(d) Cross section of $x-y$ plane

Fig 1. Body dynamics model of C.elegans

be investigated by recording its behavior and examining the internal states of the neural network. Even better, recently, dopamine was identified as a key chemical ${ }^{2}$ intermediating the distinctive gait changes observed when the worm swims in water and crawls on $\operatorname{agar}^{3,4}$. It was also revealed that dopamine-related changes are induced by mechanical stimuli ${ }^{2}$. However, for the reason of both experimental conditions and technological limits, it has been difficult to measure the amount of forces between the body and environments without considering of effects of its behavior. As a result, the input for its neural network system remains unclear.

Computer simulation provides a potential solution to this problem because the worm's thrust is generated by reaction forces based on the frictions between its body and environments in the absence of other external forces, and can be calculated using motion equations. The body dynamics model of $C$. elegans, which at first has been proposed by Niebur and Erdos 5 , was able to simulate the worm's undulatory locomotion so that a model worm could travel as fast as a real one. As friction cannot be measured from an actual worm, the friction coefficients included in the model were estimated from the friction measured between the glass fiber and agar $^{6}$. Recently, more precise frictioncoefficients in the gelatin solution and water were calculated by Berri et al. and Sznitman et al. based on the principles of fluid dynamics ${ }^{7 \sim 9}$. However, previous methods have limitations in terms of the range of environments in which they can be applied to. An algorithm to determine friction independent of environmental conditions is therefore needed to support estimation of the mechanical stimuli affecting on $C$. elegans.

In this paper, the authors propose a body dynamics model incorporating the considerations of dynamic and viscous friction. An estimation method for friction coefficients based on the model is then proposed. As this method requires only locomotion information obtained from video analysis of worms, it enables us to estimate frictions regardless of the environment and the worm's behavior. Chapter 2 describes the body dynamics model of $C$. elegans and the estimation method for environmental frictions. Chapter 3 covers verification of the proposed algorithm and reports on the results of environmental friction estimation. Chapter 4 discusses the relationship between translational force and friction force, and Chapter 5 details the conclusion and outlines future works.

\section{Materials and Methods}

When $C$. elegans moves on a solid surface such as an agar plate or swims in liquid, it is exposed to different environmental drag forces. As these forces are the basis of propulsion, different drag characteristics or strengths produce different propulsive movements. Drag force can therefore be derived by observing the motion of worms to solve dynamic problems. The following sections describe the materials and methods used to record worm motions, and highlights the algorithms used to estimate drag force.

\subsection{Strains and culture}

The $C$. elegans wild-type Bristol $\mathrm{N} 2^{10}$ and the Escherichia coli OP50 strain were obtained from the Caenorhabditis Genetics Center. Using standard methods ${ }^{10}$ worms were grown at $20^{\circ} \mathrm{C}$ on $6-\mathrm{cm}$ nontreated petri plates (IWAKI $60 \mathrm{~mm} /$ Non Treated Dish, AGC Techno Glass Co., Ltd., Shizuoka, Japan) containing $10 \mathrm{ml}$ of nematode growth medium (NGM) agar spread with E. coli (food). Well-fed worm at the young adult stage were used in all assays.

\subsection{Sample preparation}

A $6-\mathrm{cm}$ plates containing $4 \mathrm{ml}$ of NGM agar for assay were prepared on the same day of experiments. The plate for the crawling assay (plate A) was uncovered and placed on a clean bench for 


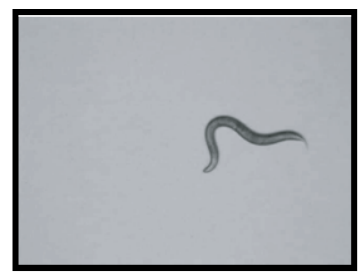

Actual worm

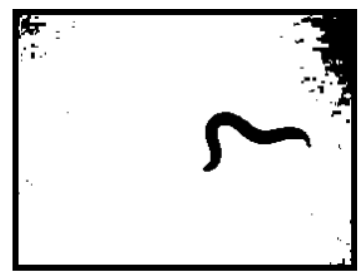

(i) Binarization

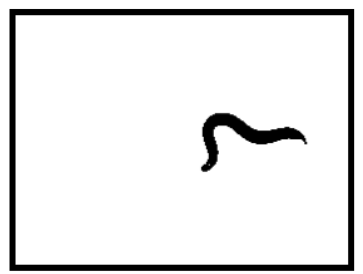

(ii) Denoising

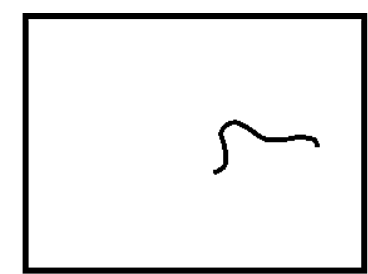

(iii) Skeletonizing

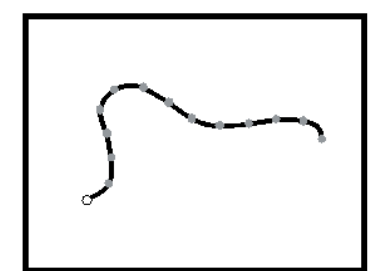

(iv) Division of body line

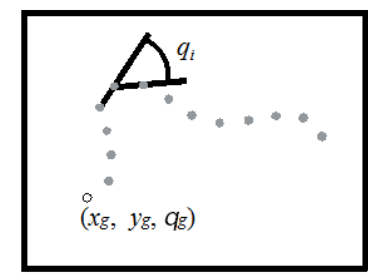

(v) - (vii) Calculation concerning with body form
Fig. 2. Image processing

approximately $1 \mathrm{~h}$ to dry up excess fluid from the surface. The plate for the swimming assay (plate B) was not dried up and was added $300 \mu \mathrm{l}$ of $\mathrm{S}$ basal buffer ${ }^{11}$. A worm was picked up from a culture plate and washed twice in a few drops of $\mathrm{S}$ basal buffer. Immediately after wash, a worm was transferred to an assay plate A or B.

\subsection{Rigid link model}

To estimate the drag forces acting between $C$. elegans and its environment, the worm's body was first approximated using the $N$ rigid link model shown in Figure 1 (c), which also illustrates its coordinate configuration. The motion of the model is restricted to the two-dimensional $x-y$ plane. The links $l_{2}-l_{\mathrm{N}-1}$ were connected with adjacent links via rotational joints to allow rotation around the z-axis, and the head link $l_{1}$ and tail link $l_{N}$ had one free end and a joint respectively connected to links $l_{2}$ and $l_{N-1}$. Using this model, the relationship between drag and motion can be derived from Newton-Euler-type motion equations as shown below.

$$
I(\boldsymbol{q})\left[\begin{array}{c}
\ddot{q}_{0} \\
\ddot{\boldsymbol{q}}
\end{array}\right]+h(\boldsymbol{q}, \dot{\boldsymbol{q}})+g(\boldsymbol{q})=\left[\begin{array}{l}
0 \\
\boldsymbol{\tau}
\end{array}\right]-\sum_{\boldsymbol{j}} \boldsymbol{J}_{j}^{T} \boldsymbol{F}_{j},
$$

$$
I_{g}(\boldsymbol{q}) \ddot{\boldsymbol{r}}_{g}+h_{g}(\boldsymbol{q}, \dot{\boldsymbol{q}})+g_{g}(\boldsymbol{q})=\sum_{j} F_{j},
$$

where $\boldsymbol{q}=\left(q_{1}, q_{2}, \ldots q_{N-1}\right)^{\mathrm{T}} \in \mathbb{R}^{N-1}$ is a vector of local bending angles, $\boldsymbol{r}=(x, y)^{\mathrm{T}}$ and $q_{0}$ denotes the tip position and its posture angle for the head link related to the global coordinate as shown in Figure 1. The terms $I(\boldsymbol{q}), I_{g}(\boldsymbol{q})$ on the left-hand side are inertial forces, $h(\boldsymbol{q}, \dot{\boldsymbol{q}}), h_{g}(\boldsymbol{q}, \dot{\boldsymbol{q}})$ are Coriolis forces and centrifugal forces, and $g(\boldsymbol{q}), g_{g}(\boldsymbol{q})$ are gravity forces. The first term $\boldsymbol{\tau} \in \mathbb{R}^{N-1}$ on the right-hand side is the torque driving each link, and the second term represents friction, where $\boldsymbol{J}_{j}^{T}$ is the Jacobean matrix of the $j$-th joint with a representative point. $\boldsymbol{F}_{j}=\left(f_{j, t}, f_{j, n}\right)^{\mathrm{T}}$ represents the drag force acting on the gravity point of each joint, and can be described in arbitrary form depending on the surrounding environment. In this study, drag force was modeled using the properties of Coulomb friction and viscous drag in a Newtonian fluid as follows:

$$
f_{j, d}=-\mu_{d} \frac{v_{j, d}}{\left|v_{j, d}\right|}-\eta_{d} v_{j, d},
$$

where $d \in(t, n)$ denotes the tangential and normal directions related to the link, $v_{j, d}$ are the velocities at the gravity point of link $j, \mu_{d}$ is the coefficient of Coulomb friction, and $\eta_{d}$ is the viscous drag coefficient.

Equation (2) indicates that the drag coefficients and torque $\boldsymbol{\tau}$ driving each joint can be derived from local bending angles and the related velocities and accelerations $(\boldsymbol{q}, \dot{\boldsymbol{q}}, \ddot{\boldsymbol{q}})$ and translational acceleration $\ddot{\boldsymbol{r}}_{g}$, which can be determined from video analysis. Based on this equation, an algorithm for friction coefficient estimation is proposed below.

\subsection{Friction estimation algorithm}

The motion equations (1) and (2) can be solved for drag coefficients given the motion information $(\boldsymbol{q}, \dot{\boldsymbol{q}}, \ddot{\boldsymbol{q}}$, $\ddot{\boldsymbol{r}}_{g}$ ). Among these values, the local bending angle $\boldsymbol{q}$ and the position $\boldsymbol{r}_{g}$ can be determined by recording the motion of a worm and analyzing the resulting video, and the related time derivations can be used to determine velocity and acceleration. The information obtained can then be used to solve the motion equations for the drag coefficients in relation to each video. However, there are two problems in the implementation of this calculation.

Firstly, it is an ill-posed problem because only $N+2$ coupled equations can be derived from Equations (1) 
and (2), while there are four unknown drag coefficients plus $N$-1 unknown torque values $\left(\boldsymbol{\tau} \in \mathbb{R}^{N-1}\right)$. To solve this problem, the proposed algorithm involves simultaneous analysis of two different motions of a worm placed in the same environment in which identical drag coefficients are assumed. In this manner, the number of unique coupled equations can be doubled without increasing the number of unknown drag coefficients.

Secondly, the drag coefficients calculated from only one sampling can be significantly affected by video analysis error. To reduce this impact, the proposed algorithm involves the solution of a motion equation for $\ddot{\boldsymbol{r}}_{g}$ and $q_{0}$ for the time duration $T$ with different drag coefficients. The coefficients that generate the paths closest to those of the actual worm are then explored by minimizing the following evaluation function:

$E=\frac{1}{2 l\left(t_{\max }+1\right)} \sum_{t=0}^{T} \sum_{i=1}^{l} \sum_{j=1}^{2}\left\|x_{i}^{j}(t)-\boldsymbol{r}_{i}^{j}(t)\right\|$,

Where $\boldsymbol{x}_{i}^{j}$ represents the position of the $i$-th evaluation point on the worm, $\boldsymbol{r}_{i}^{j}$ represents the corresponding position on the rigid-link body model, and subscript $j$ denotes an individual worm.

The proposed algorithm is applied to estimate the drag coefficients for a worm swimming in liquid and crawling on an agar surface. The proposed algorithm consists of four blocks as outlined below.

\section{Block 1: Video analysis}

To analyze the body form of $C$. elegans in crawling and swimming, the worm on an assay plate (A for crawling and $\mathrm{B}$ for swimming) was video-recorded using a digital camera EXILIM EX-F1 (CASIO Computer Co., Ltd., Tokyo, Japan) mounted on a stereomicroscope for approximately 10 seconds at 300 frames per second. The resolution of each frame was 512 x 384 pixels. Distinct worms picked up from a culture plate were used for crawling assays and swimming assays. The body form in crawling and swimming of $C$. elegans was analyzed off-line based on the previous method ${ }^{12}$ as shown in Figure 2. Briefly, each frame of the video was processed using the following procedures: (i) binarization, (ii) denoising, (iii) skeletonizing, and (iv) division of body line into the 13 parts. After image processing, time-series data concerning with the body form were obtained using the following procedures: (v) length-scale calibration, (vi) acquisition of the $x$ - and $y$-coordinates of each point on the body, and (vii) calculation of values concerning with the body form such as the relative angle between adjacent dividing points. Video analyses described above were carried out using the following software: Wriggle Tracker (Library Co. Ltd., Tokyo, Japan) for procedures (i)-(iv) and Move-tr/2D (Library Co. Ltd., Tokyo, Japan) for procedures (v)-(vii).

\section{Block 2: Selection of drag coefficient}

In this block, a set of coefficients is selected in three phases as described below.

\section{[Phase 1]}

In Phase 1, drag coefficients are selected from the set $\left(\mu_{n}, \mu_{t}, \eta_{n}, \eta_{t}\right) \in\left\{\left(N_{1} \Delta \mu, N_{2} \Delta \mu, N_{3} \eta, N_{4} \eta\right) \mid\right.$ $\left.N_{1}, N_{2}, N_{3}, N_{4}=0,1, \cdots, N^{\max }\right\}$ based on iterative alteration of $N_{1}, N_{2}, N_{3}, N_{4}$.

After the search for all combinations is complete, $d_{1} \%$ of the drag coefficients $\left(\mu_{n}^{E_{1, a}}, \mu_{t}^{E_{1, a}}, \eta_{n}^{E_{1, a}}, \eta_{t}^{E_{1, a}}\right)$ with smaller evaluation values (Equation (4) are selected, and the iteration proceeds to Phase 2 , where $a=1,2, \ldots, d_{1}\left(N^{\max }+1\right)^{4} / 100$.

\section{[Phase 2]}

In Phase 2, better drag coefficients are sought from the set selected in Phase 1. The search is carried out with the set of $\left(\mu_{n}^{E_{1, a}}+N_{1}\left(\frac{\Delta \mu}{2}\right), \mu_{t}^{E_{1, a}}+N_{1}\left(\frac{\Delta \mu}{2}\right)\right.$, $\left.\eta_{t}^{E_{1, a}}+N_{1}\left(\frac{\Delta \eta}{2}\right), \eta_{t}^{E_{1, a}}+N_{1}\left(\frac{\Delta \eta}{2}\right)\right) \quad$ where $\quad N_{1 \sim 4}=$ $\{-1,0,1\}$. After the search for all combinations is complete, $d_{2} \%$ of the drag coefficients $\left(\mu_{n}^{E_{2, b}}, \mu_{t}^{E_{2, b}}, \eta_{n}^{E_{2, b}}, \eta_{t}^{E_{2, b}}\right)$ with smaller evaluation values (Equation (4)) are selected, and the iteration proceeds to Phase 3, where $b=1,2, \ldots, 3 d_{1} d_{2}\left(N^{\max }+1\right)^{4} / 10000$.

\section{[Phase 3]}

In this phase, the generalized reduced gradient method $^{13}$ is applied for the selection of drag coefficients $\operatorname{using}\left(\mu_{n}^{E_{2, b}}, \mu_{t}^{E_{2, b}}, \eta_{n}^{E_{2, b}}, \eta_{t}^{E_{2, b}}\right)$ as initial values so that the friction coefficients with the smallest evaluation values are calculated.

\section{Block 3: Calculation of Motion Equations}

In this block, calculation for the path of two rigidlink body models in the time span $T \mathrm{~s}$ is performed with the friction coefficients selected in Block 2. Calculation to solve Equations (1) and (2) for $q_{0}, \boldsymbol{r}$ is carried out 
Table 1 Parameters used in simulation

\begin{tabular}{|l|c||c|c|}
\hline$t_{\max }$ & $5 \mathrm{~s}$ & $\Delta \mu$ & $1.0 \times 10^{-1}$ \\
\hline$l$ & 3 & $\Delta \eta$ & $1.0 \times 10^{-3}$ \\
\hline$m$ & $3.84 \times 10^{-7} \mathrm{~g}$ & $d_{1}$ & $1.0 \%$ \\
\hline$N^{\max }$ & 10 & $d_{2}$ & $0.2 \%$ \\
\hline
\end{tabular}

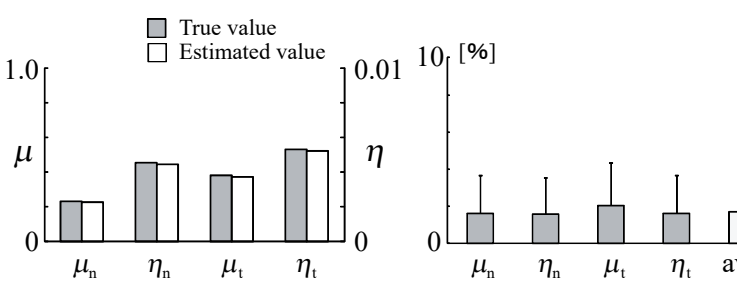

(a) Result of $F_{1}$ (b) Percentage errors

Fig. 3. Estimated results of friction coefficients

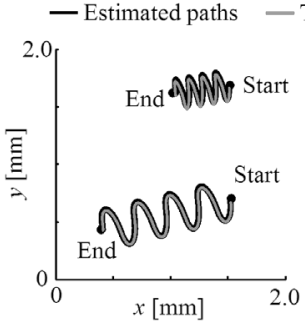

(a) Result of F

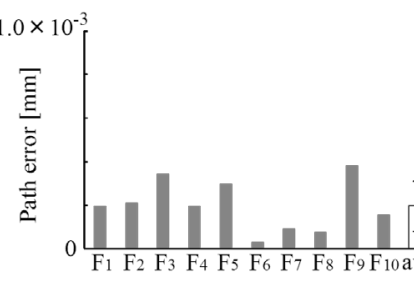

(b) Path errors
Fig. 4. Estimated paths

using ADAMS (MSC Software Corporation, Tokyo, Japan) multi-body dynamics simulator.

\section{Block 4: Evaluation of Drag Coefficients}

In Block 4, evaluation is performed for drag coefficients by substituting the paths calculated in Block 3 into an evaluation function (Equation (4)), and the iteration returns to Block 2.

\section{Simulation Experiments}

As a way of verifying the performance of the proposed body dynamics model and the estimation algorithm for friction coefficients, testing was performed to determine whether the algorithm enabled estimation of artificially preset friction coefficients. Such coefficients were then estimated for worms crawling on agar and swimming in a drop of water.

\subsection{Verification}

The locomotion of the body model was simulated using Equations (1) and (2) with artificially preset friction coefficients and time-dependent joint angles.

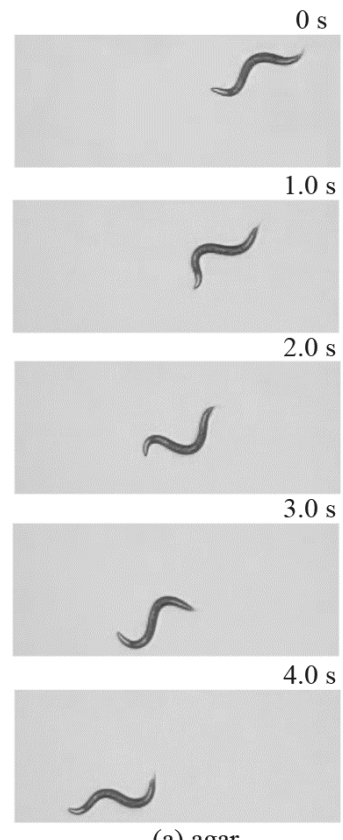

(a) agar

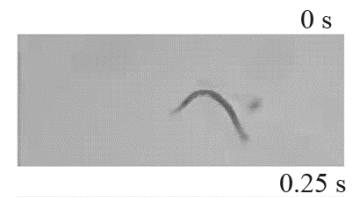

$0.25 \mathrm{~s}$

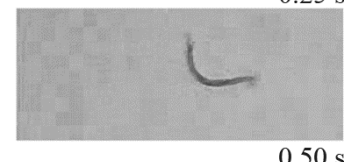

$0.50 \mathrm{~s}$

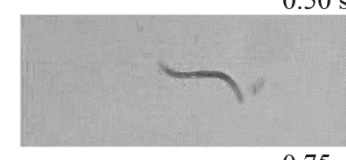

0.75
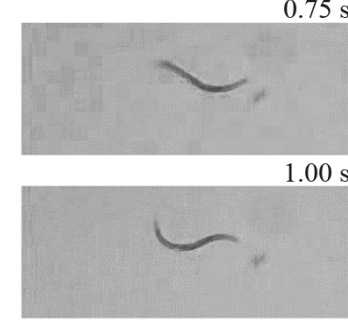

(b) Water
Fig. 5. Locomotion of C. elegans

The paths determined from this simulation were then used with the proposed algorithm for friction coefficient estimation. Finally, the errors observed between the preset and estimated friction coefficients were calculated to verify the performance of the algorithm. Here, time-dependent joint angles with the following four patterns were chosen: $q_{i+1}-q_{i}=0.4 \sin (5 t-$ $0.6 i), 0.6 \sin (5 t-0.6 i), 0.4 \sin (5 t-0.6 i)+0.1$, $0.6 \sin (5 t-0.6 i)+0.1$ which are respectively denoted as Locomotion 1 4. In addition, 10 sets of friction coefficients $\left(F_{1} \sim F_{10}\right)$ were chosen as uniform random numbers in the range of $[0,1]$ for dynamic friction coefficients and $[0,0.01]$ for viscous friction coefficients. The locomotion pair of 1 and 2 was simulated with the friction coefficients of $F_{1} \sim F_{5}$ and the other pair (Locomotion 3 and 4) was simulated with the friction coefficients of $F_{5} \sim F_{10}$. The parameters of the body dynamics model and the proposed estimation algorithm are shown in Table 1.

Figure 3 shows the friction coefficient estimation results and the related percentage errors against the preset values. Figure 3(b) shows that the average percentage errors were less than $2 \%$ with a standard deviation of $2 \%$. Figure 4 shows an example result for the paths of Joint 7 as well as average path errors for all simulations performed. It can be seen that the average error was $1.9 \times 10^{4} \pm 1.1 \times 10^{-4} \mathrm{~mm}$, which indicates that the proposed algorithm is capable of generating 


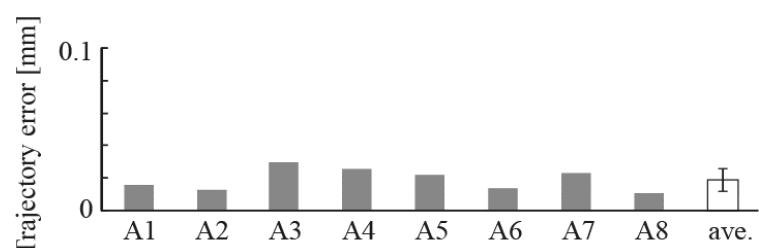

Fig. 6. Resulted value of evaluation function of crawling simulation

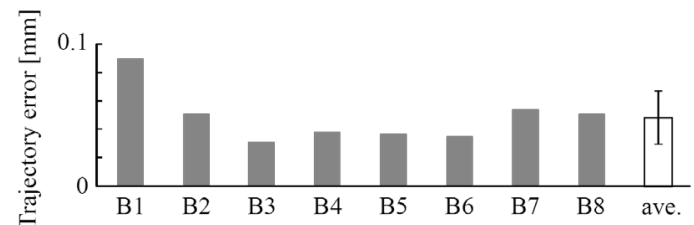

Fig. 7. Resulted value of evaluation function of swimming simulation

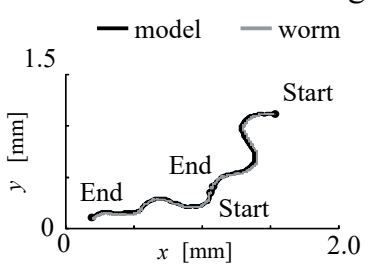

(a) Paths of crawling

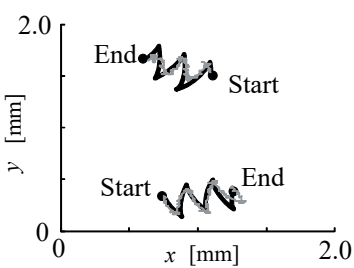

(b) Paths of swimming
Fig. 8. Paths of seventh joint

locomotion values with errors smaller than $2.9 \times$ $10^{-2} \%$ in relation to the worm's length.

\subsection{Estimation of friction coefficients of actual worms}

Friction coefficients for actual worms were estimated using video of their movement on agar and in a drop of water (Figures 5). Each of the time-dependent joint angles determined from image processing was smoothed using a second-order Butterworth low-pass filter (high cutoff frequency: $5 \mathrm{~Hz}$ ) for denoising. The analysis times for crawling and swimming were respectively set as $t_{\max }=4 \mathrm{~s}, 1 \mathrm{~s}$, and the other parameters were the same as those used in the verification simulation.

Figures 6 and 7 show the values of evaluation function (Equation (4)) acquired from swimming and crawling simulation, respectively. Here, the evaluation function represents the errors between the paths of the models and that of the actual worms. The average path error of crawling simulation was about $0.02 \mathrm{~mm}$ ( $2 \%$ of the worm's length), and the average path error of swimming simulation was $0.05 \mathrm{~mm}$ (4\% of the worm's length). Figure 8 shows the paths of seventh joint in crawling and swimming simulation. These outcomes indicate that each of the simulated path fitted worm paths well. Figures 9 and 10 show friction coefficients estimated from eight video images for crawling and eight for swimming, with the numbers under each picture distinguishing individual worms. It can be seen that the estimated friction coefficients differed greatly even for the same environments. In particular, in Figure 9(A1) and (A3) representing situations in which friction coefficients were estimated from the same worm crawling on the same agar plate, the viscous friction coefficient for the normal direction shown in (A1) was much larger than that shown in (A3). The next section discusses this friction coefficient variability.

\section{Discussion}

This section discusses the cause of variances found in the estimated friction coefficients $\mathrm{A} 1, \mathrm{~A} 2$ and $\mathrm{A} 3$ (Fig. 10) or the same worm on the same agar plate. 


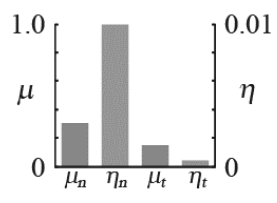

(A1) Worm 1

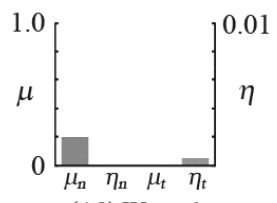

(A3) Worm 1

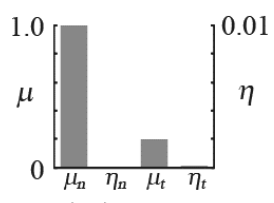

(A5) Worm 3

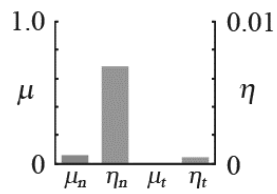

(A7) Worm 5

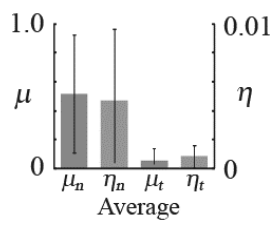

Fig. 9. Estimated friction coefficients (agar)

To compare the effects of different friction coefficients adopted for the same joint motion, the timedependent joint angles $\boldsymbol{q}^{\mathrm{Al}}(t)$ extracted from the locomotion of A1 were substituted into Equations (1) and (2), and paths with the friction coefficients of A2 and A3 were calculated. Figure 11 shows paths resulting from simulation under the time-dependent joint angles $q^{\mathrm{A} 1}(t)$ and the friction coefficients $\mathrm{A} 2$ and A3. The errors observed between the actual and simulated worm's paths are also shown in Figure 12(b). These results indicate that the average path error was about $4 \%$ of the worm's length even when different friction coefficients (A1, A2 and A3) were used. Figure 12(a) highlights the friction force at each link accumulated at the point of the head tip. The right-hand side of Equation (2) shows this calculation. From the figure, it can be seen that the total friction generated from friction coefficient A1 showed a strong correlation and similar values to that generated from friction coefficients A2 and $\mathrm{A} 3$. As the total friction reactive forces correspond

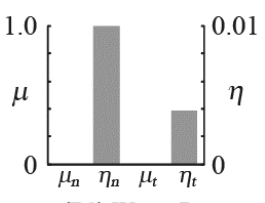

(B1) Worm 7

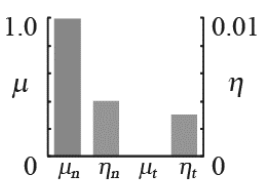

(B3) Worm 9
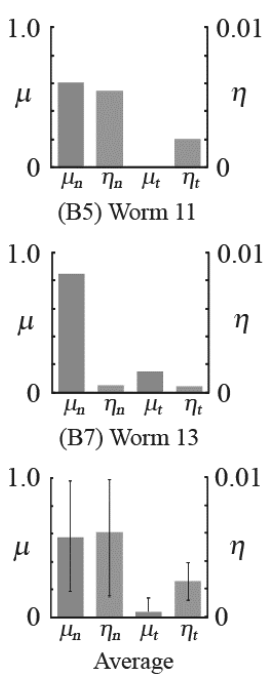

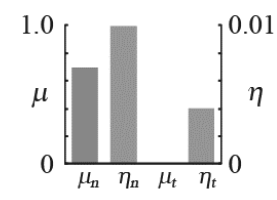

(B2) Worm 8
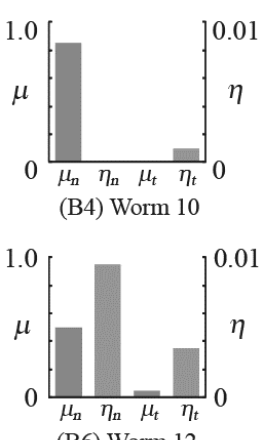

(B6) Worm 12

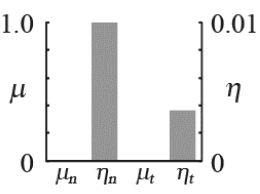

(B8) Worm 14
Fig. 10. Estimated friction coefficients (water)

to the propulsion forces, this result indicates that the proposed algorithm with an evaluation function defined by path errors can be used to estimate propulsion force.

In the previous studies regarding the dynamic modeling of $C$. elegans, it was reported that a worm's path can be determined from the ratio between the normal and tangential friction coefficient. ${ }^{5,7}$ Indeed, the friction coefficient ratio is a dominant factor in determining worm paths. However, according to the simulation performed here, identical locomotion cannot be generated if the same ratio but different friction coefficients are adapted. Figure 13 shows locomotion paths observed when the dynamic friction coefficients were kept at a constant ratio $\left(2 \mu_{\mathrm{t}}=\mu_{\mathrm{n}}\right)$ and the viscous friction coefficient was set to $0\left(\eta_{\mathrm{t}}=\eta_{\mathrm{n}}=0\right)$. The results demonstrate that there were path differences depending on the value of $\mu_{\mathrm{n}}$, even though the dynamic friction coefficients were kept at a constant ratio. This suggests that if image processing on actual worms can be carried out with a high degree of accuracy, friction coefficients 


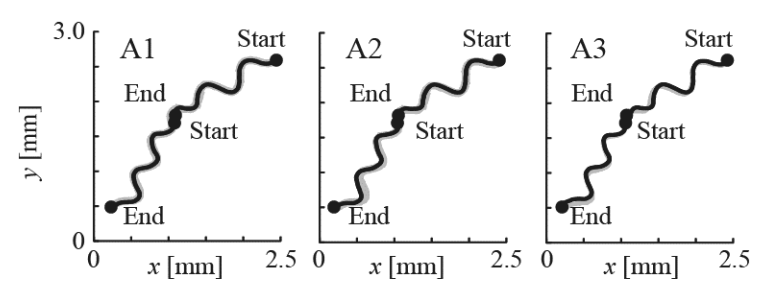

(a) Paths under different friction coefficients

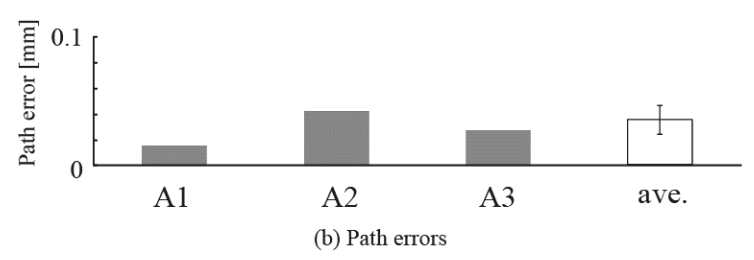

Fig. 11. Comparison of trajectories (agar)

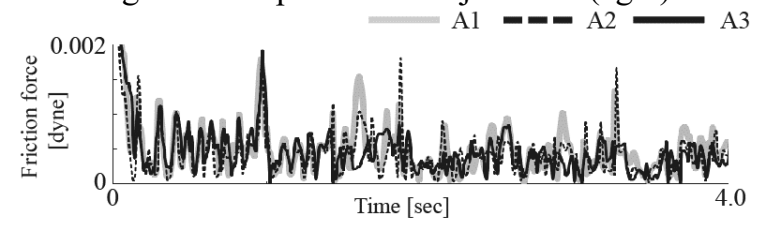

(a) Comparison of friction force

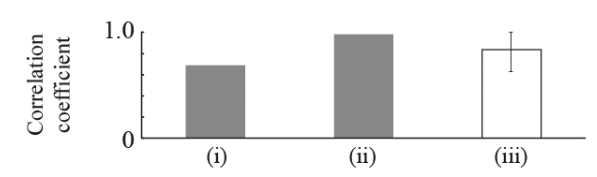

(b) Correlation coefficients: (i) Between $\mathrm{Al}$ and $\mathrm{A} 2$ (ii) Between A1 and A3, (iii) Average

Fig. 12. Comparison of friction force

can be uniquely determined because verification simulation under artificially preset configurations as described in Section 3.1 enabled the estimation of friction coefficients close to actual values with an acceptable level of accuracy.

Finally, the effect of friction in different environments was quantified. Figure 14 shows the average RMS value of the total friction forces as calculated from the crawling and swimming simulation results. The result of a $t$-test to determine the RMS between crawling and swimming showed a significant difference between the two $(p<0.001)$. Fig. 15 indicates that worms experience stronger friction forces when swimming than when crawling. Using results obtained from the proposed estimation algorithm may allow to clarify the underlying mechanisms of gait control ${ }^{8}$ at the friction level.

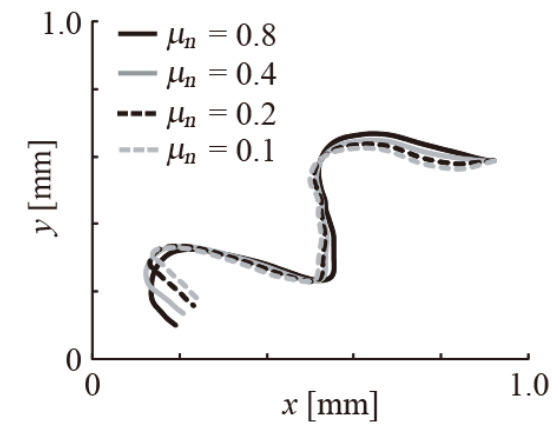

Fig. 13. Paths under the same friction coefficient

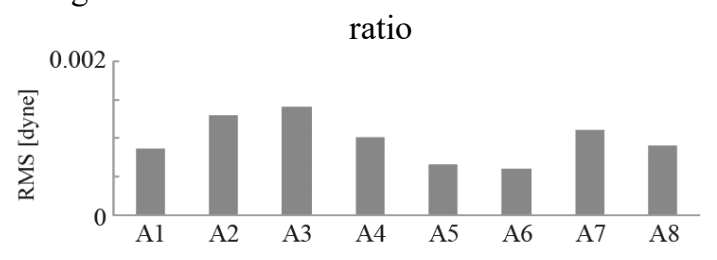

(a) RMS of friction force on agar plate

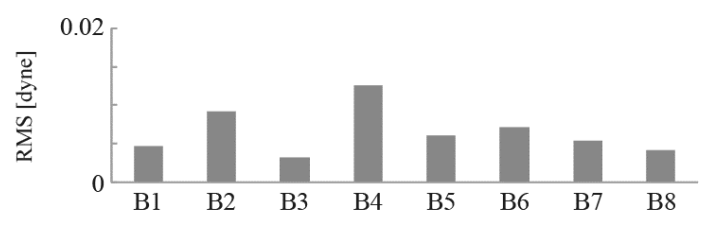

(b) RMS of friction force in water drop

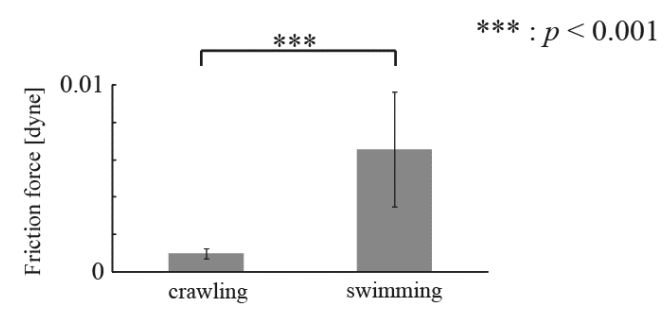

(c) Average of RMS on agar plate and in water drop

Fig. 14. Average RMS

\section{Conclusion and Future Works}

This paper proposed a method for estimating friction coefficients based on a body dynamics model and video images of $C$. elegans. The study verified that the proposed algorithm can be used to estimate friction coefficients under artificially preset configurations, and the results of experiments using actual worms confirmed that the model can track worm paths with small errors. The algorithm can be used to estimate true friction coefficient values under ideal noise-free conditions. Although estimation results were affected by noise contamination from image processing, the propulsion forces affecting locomotion paths could be estimated. 
In future works, the authors plan to improve the robustness of the proposed method to support the calculation of time-dependent friction changes acting on worms, and will analyze the underlying mechanisms of gait control against environmental factors.

\section{Acknowledgements}

The authors appreciate to Dr. Y. Hattori and Mr. S. Nishikawa for valuable discussions. This work was supported by JSPS KAKENHI Grant Numbers $15 \mathrm{H} 03950$ and 20115010.

\section{References}

1. J. G. White, E. Southgate, J. N. Thomson and S. Brenner, The Structure of The Nervous System of Nematode Caenorhabditis elegans, Philosophical Transactions of the Royal Society of London, 314 (1986) 1-340.

2. A. Vidal-Gadea, S. Topper, L. Young, A. Crisp, L. Kressin, E. Elbel, T., Maples, M. Brauner, K. Erbguth, A. Axelrod, A. Gottschalk, D. Siegel and J. T. PierceShimomura, Caenorhabditis elegans selects distinct crawling and swimming gaits via dopamine and serotonin, Proc. Natl. Acad. Sci. U.S.A. 108(42) (2011) 17504-17509.

3. J. Gray and H. W. Lissmann, The Locomotion of Nematodes, J. Exp. Biol. 41 (1964) 135-154.

4. J. Korta, D. A. Clark, C. V. Gabel, L. Mahadevan and A. D. T. Samuel, Mechanosensation and mechanical load modulate the locomotory gait of swimming C. elegans, $J$. Exp. Biol. 210 (2007) 2383-2389.

5. E. Niebur and P. Erdos, Theory of the locomotion of nematodes, Biophys. J. 60 (1991) 1132-1146.

6. H. R. Wallace, Wave formation by infective larvae of the plant parasitic nematode Meloidogyne javanica, Nematology. 15 (1969) 65-75.

7. S. Berri, J. H. Boyle, M. Tassieri, I. a Hope and N. Cohen, Forward locomotion of the nematode $C$. elegans is achieved through modulation of a single gait, HFSP $J$. 3(3) (2009) 186-193.

8. J. T. Pierce-Shimomura, B. L. Chen, J. J. Mun, R. Ho, R. Sarkis and S. L. McIntire, Genetic analysis of crawling and swimming locomotory patterns in C. elegans, Proc. Natl. Acad. Sci. U.S.A. 105(52) (2008) 20982-20987.

9. J. Sznitman, X. Shen, P. K. Purohit and P. E. Arratia, The Effects of Fluid Viscosity on the Kinematics and Material Properties of $C$. elegans Swimming at Low Reynolds Number, Exp. Mech. 50(9) (2010) 1303-1311.

10. S. Brenner, The Genetics of Caenorhabditis elegans, Genetics 77(1) (1974) 71-94.

11. E. R. Sawin, R. Ranganathan, and H. R. Horvitz, $C$. elegans Locomotory Rate is Modulated by the Environment through a Dopaminergic Pathway and by Experience through a Serotonergic Pathway, Neuron, 26(3) (2000) 619-631.

12. Y. Hattori, M. Suzuki, Z. Soh, Y. Kobayashi and T. Tsuji, Theoretical and evolutionary parameter tuning of neural oscillators with a double-chain structure for generating rhythmic signals, Neural Comput., 24 (2012) 635-675

13. G. A. Gabriele and K. M. Ragsdell, The Generalized Reduced Gradient Method: A Reliable Tool for Optimal Design, J. Eng. Ind., 99(2) (1976) 394-400. 